\title{
Chiral phase transitions of helical matter
}

\author{
Shen-Song Wan®, Gianluca Giuliani, ${ }^{\dagger}$ and Marco Ruggieri®* \\ School of Nuclear Science and Technology, Lanzhou University, \\ 222 South Tianshui Road, Lanzhou 730000, China
}

(Received 24 December 2020; accepted 4 May 2021; published 25 May 2021)

\begin{abstract}
We study the thermodynamics of helical matter, namely quark matter in which a net helicity $n_{H}$ is in equilibrium. Interactions are modeled by the renormalized quark-meson model with two flavors of quarks. Helical density is described within the grand-canonical ensemble formalism via a chemical potential $\mu_{H}$. We study the transitions from the normal quark matter and hadron gas to the helical matter, drawing the phase diagram at zero temperature. We study the restoration of chiral symmetry at finite temperature and show that the net helical density softens the transition, moving the critical end point to lower temperature and higher baryon chemical potential. Finally, we discuss briefly the effect of a rigid rotation on the helical matter, in particular on the fluctuations of $n_{H}$, and show that these are enhanced by the rotation.
\end{abstract}

DOI: 10.1103/PhysRevD.103.094030

\section{INTRODUCTION}

The phase diagram of quantum chromodynamics (QCD) in the temperature $T$ and baryon chemical potential $\mu$ plane is one of the most active research subjects in modern highenergy physics. At $\mu=0$, first-principles lattice QCD calculations show that QCD matter experiences a crossover from a low-temperature confined phase, in which chiral symmetry is spontaneously broken, to a high-temperature phase, in which chiral symmetry is approximately restored [1-5]. It is not possible to define uniquely a critical temperature for the restoration of chiral symmetry: it is more appropriate to define a pseudocritical region centered on a pseudocritical temperature $T_{c}$ that is a range of temperatures in which several physical quantities experience substantial changes. The (pseudo)critical temperature is found to be $T_{c} \approx 150 \mathrm{MeV} \approx 10^{12} \mathrm{~K}$.

Despite the amazing results obtained by lattice QCD in recent years, it is not possible to perform reliable simulations of QCD with three colors at large $\mu$. For this reason, the use of effective models for studying the phase transitions of $\mathrm{QCD}$ at $\mu \neq 0$ is necessary. In this context the quark-meson (QM) model is very popular due to its renormalizability [6-12]. The aforementioned chiral crossover is reproduced by the QM model, with $T_{c}$ in the same ballpark of the QCD one. At $\mu \neq 0$ the smooth crossover of the QM model

\footnotetext{
ruggieri@lzu.edu.cn

gianluca@lzu.edu.cn
}

Published by the American Physical Society under the terms of the Creative Commons Attribution 4.0 International license. Further distribution of this work must maintain attribution to the author(s) and the published article's title, journal citation, and DOI. Funded by SCOAP ${ }^{3}$. becomes a first-order phase transition, suggesting a critical end point (CEP) in the $\mu-T$ plane at which the crossover becomes a second-order phase transition with divergent susceptibilities, marking the separation between the crossover on the one hand and a first-order line on the other hand. Similar conclusions have been obtained within the NambuJona-Lasinio model [13-19]. Moreover, in recent years the picture of the QM model has been strengthened by the inclusion of critical fluctuations via the functional renormalization group [20-23].

Besides $\mu$, it has been recently suggested that another chemical potential, $\mu_{H}$, conjugated to the helical density $n_{H}$, can be of interest for the structure of QCD; see [24-29]. Matter with $n_{H} \neq 0$ is called helical matter and is opposed to normal quark matter in which baryonic density $n_{B} \neq 0$ and $n_{H}=0$. The very basic idea of helical matter is that helicity is a conserved quantities for free massless as well as massive fermions; therefore, if a net helicity is formed in a system made of free quarks, a chemical potential $\mu_{H}$ can be introduced that is conjugated to $n_{H}$, similarly to the baryon chemical potential that is conjugated to the baryonic density. For the quark-gluon plasma produced in highenergy nuclear collisions, lumps of matter with $\left\langle n_{H}^{2}\right\rangle \neq 0$ can be formed thanks to event-by-event fluctuations, and the helical density is approximately conserved because helicity-changing processes take place on a timescale that typically is larger than the lifetime of the quark-gluon plasma itself [30]. The fact that matter with helical density can be of relevance for high-energy nuclear collisions has been discussed very recently [24-29]; in these works, it has been argued that spin dynamics is slow enough in the collisions, suggesting that helicity can be considered as approximately conserved for the entire quark-gluon plasma lifetime and be one source of the observed polarization of the $\Lambda$ particles. 
We report on helical matter both at zero and finite temperature. $\mu_{H}$ does not introduce additional divergences; therefore, the renormalization of the vacuum quark loop can be done in the standard manner [6-10]. We discuss the transition from the hadron gas to normal quark matter and helical matter. We focus on the baryonic and the helical density susceptibility. We show that close to the phase transition, it is possible to distinguish between normal and helical matter not only in terms of the densities, but also in terms of the susceptibilities that indeed measure the fluctuations of the conserved quantities. Our results have some overlap with Ref. [24], and we agree with that reference whenever we compute similar quantities. One of our conclusions is that helical density makes the chiral phase transition softer. Thus, the formation of lumps of helical matter in the quark-gluon plasma produced in highenergy nuclear collisions can impact the observables for the critical end point, since for regions in which $\left\langle n_{H}^{2}\right\rangle \neq 0$ the critical end point would move to larger values of the baryon chemical potential and smaller temperatures.

We complete our study by suggesting a coupling of the fluctuations of $n_{H}$ and the angular velocity $\omega$ of a rigid rotation. Rotation has been considered in the context of hot quark matter several times in recent literature; see [25,31-42], and references therein. This is the first time in which rotation is considered within helical matter; therefore, we limit ourselves to the simplest implementation of rotation, namely that of an unbounded system, and we study only quark matter in proximity of the rotation axis. We illustrate, by using an ideal gas as an example, that rotation links to the fluctuations of the helical density and enhances them. We compute analytically the relation between the helical susceptibility and $\omega$ in the case of a massless gas. Then we show how this link appears within the QM model. We think that also this part of the study motivates the relevance of helical matter for the quarkgluon plasma produced in high-energy nuclear collisions, due to the vorticity produced in the collisions [43-51] that enhances the fluctuations of $n_{H}$.

The plan of the article is the following. In Sec. II we formulate the QM model with a helical chemical potential and the renormalization of the vacuum term. In Sec. III we discuss the phase diagram at $T=0$. In Sec. IV we present the results at finite temperature. In Sec. V we discuss the enhancement of the fluctuations of helical density in a rotating medium. Finally, in Sec. VI we summarize our conclusions and an outlook of possible future projects. We use the natural units system $\hbar=c=k_{B}=1$ throughout this article.

\section{THE QUARK-MESON MODEL FOR HELICAL MATTER}

In this section we summarize the QM model that we use in our study. We firstly define the helical chemical potential $\mu_{H}$ that we couple to quarks beside the standard quark number chemical potential $\mu$. Then we define the Lagrangian density of the model. Finally, we specify the thermodynamic grand potential and its renormalization procedure.

\section{A. Helical chemical potential}

We begin with a free, massive Dirac fermion. Helicity is defined, for momentum eigenstates with eigenvalues $\boldsymbol{p}$, as the projection of spin $s$ over the direction of momentum $\boldsymbol{p}$, that is,

$$
h=\frac{\boldsymbol{s} \cdot \boldsymbol{p}}{|\boldsymbol{p}|},
$$

where $s_{i}=\frac{1}{2} \varepsilon_{0 i j k} \Sigma^{j k}$ is the spin operator with $\Sigma^{\mu \nu}=\frac{i}{4}\left[\gamma^{\mu}, \gamma^{\nu}\right]$. Helicity can take the values $h= \pm 1 / 2$ only; for convenience, we rescale the helicity operator by a factor of 2 as $\eta=2 h$ and get $\eta \psi=\aleph \psi$. Thus, the eigenvalues $\boldsymbol{\aleph}$ are now \pm 1 . Helicity is relevant, since it is conserved for a free Dirac fermion, and can be used beside momentum to label the energy eigenstates. The helicity operator (1) is nonlocal in coordinate space, due to the $1 /|\boldsymbol{p}|$ : however, a proper system of eigenfunctions can be chosen to take the trace for the computation of the thermodynamic potential, namely the standard momentum and helicity eigenfunctions: with this choice the nonlocality of $h$ is harmless and states can be labeled by the helicity eigenvalue [36].

The aim of our study is to consider the effects of helicity on the QCD phase diagram: helicity is a conserved quantity for a free massive fermion; therefore, it is possible to introduce a chemical potential $\mu_{H}$ that is coupled to the net helicity of the system in equilibrium, $n_{H}=N_{+}-N_{-}$, where $N_{ \pm}$denotes the number of particles with $\boldsymbol{\aleph}= \pm 1$. For the Dirac field the relevant operator is

$$
\hat{N}_{h}=\bar{\psi} \gamma^{0} \eta \psi
$$

analogous to the quark number operator

$$
\hat{N}=\bar{\psi} \gamma \gamma^{0} \psi
$$

It is an obvious fact that in hot quark matter, as the one produced in high-energy nuclear collisions, the helicity of quarks can be changed by microscopic processes even though regions with $\left\langle n_{H}^{2}\right\rangle \neq 0$ are formed by event-byevent fluctuations; however, these processes are known to happen for timescales larger than the typical lifetime of the fireball [30]; therefore, for the purpose of describing the matter produced in the collisions, net helicity is fairly conserved and it is possible to introduce a chemical potential $\mu_{H}$ conjugated to $\hat{N}_{h}$ in the model.

The Lagrangian density of a free, massive Dirac fermion with both helical and number chemical potential $\mu$ in momentum space is

$$
\mathcal{L}=\bar{\psi}\left(\not p+\mu_{H} \gamma^{0} \eta+\mu \gamma^{0}+m\right) \psi .
$$


The poles of the propagator in momentum space define the energy spectrum:

$$
p_{0, \aleph}^{(s)}(\boldsymbol{p})=\sqrt{\boldsymbol{p}^{2}+m^{2}}-s \mu-\aleph \mu_{H},
$$

with $s= \pm 1$ and $\aleph= \pm 1$.

\section{B. Lagrangian density of the quark-meson model}

The QM model consists of a mesons and quarks that interact via an $O(4)$-invariant local interaction term. The meson part of the Lagrangian density of the QM model is

$$
\begin{aligned}
\mathcal{L}_{\text {mesons }}= & \frac{1}{2}\left(\partial^{\mu} \sigma \partial_{\mu} \sigma+\partial^{\mu} \pi \partial_{\mu} \pi\right) \\
& -\frac{\lambda}{4}\left(\sigma^{2}+\pi^{2}-v^{2}\right)^{2}-h \sigma,
\end{aligned}
$$

where $\pi=\left(\pi_{1}, \pi_{2}, \pi_{3}\right)$ corresponds to the pion isotriplet field. This Lagrangian density is invariant under $O(4)$ rotations. On the other hand, as long as $v^{2}>0$ the potential develops an infinite set of degenerate minima. Among these minima, we choose the ground state to be the one corresponding to

$$
\langle\boldsymbol{\pi}\rangle=0, \quad\langle\sigma\rangle \neq 0,
$$

since $\langle\boldsymbol{\pi}\rangle \neq 0$ would correspond to a spontaneous breaking of parity which is not observed in QCD, unless an isospin chemical potential is introduced; see [52] for a review. The ground state specified in Eq. (7) breaks the $O(4)$ symmetry down to $O(3)$ since the vacuum is invariant only under the rotations of the pion fields. The term

$$
\mathcal{L}_{\text {mass }}=h \sigma
$$

in Eq. (6) is responsible of the explicit breaking of chiral symmetry, where $h=F_{\pi} m_{\pi}^{2}$.

The quark sector of the QM model is described by the Lagrangian density

$$
\mathcal{L}_{\text {quarks }}=\bar{\psi}\left(i \partial_{\mu} \gamma^{\mu}-g\left(\sigma+i \gamma_{5} \pi \cdot \tau\right)\right) \psi,
$$

where $\tau$ are Pauli matrices in the flavor space. Quarks get a constituent mass because of the spontaneous breaking of the $O(4)$ symmetry in the meson sector:

$$
M_{q}=g\langle\sigma\rangle .
$$

In this study we limit ourselves to the mean field approximation in which $\sigma=\langle\sigma\rangle$ and $\pi=\langle\pi\rangle$; therefore, from now on anytime we write down a meson field we have in mind its expectation value and we will skip the \langle\rangle for the sake of notation. Finally, the Lagrangian density of the model is given by

$$
\mathcal{L}_{\mathrm{QM}}=\mathcal{L}_{\text {quarks }}+\mathcal{L}_{\text {mesons }}
$$

\section{Thermodynamic potential}

The mean field effective potential of the QM model at zero temperature is given by

$$
\Omega=U+\Omega_{q}+\Omega_{T},
$$

where

$$
U=\frac{\lambda}{4}\left(\sigma^{2}+\pi^{2}-v^{2}\right)^{2}-h \sigma
$$

is the classical potential of the meson fields as it can be read from Eq. (6) while $\Omega_{q}+\Omega_{T}$ corresponds to the sum of the vacuum and thermal contributions of the quarks.

Firstly we analyze the vacuum term

$$
\Omega_{q}=-2 N_{c} N_{f} \int \frac{d^{3} p}{(2 \pi)^{3}} E_{p},
$$

with $E_{p}=\sqrt{p^{2}+M_{q}^{2}}$ and $M_{q}$ denotes the constituent quark mass defined in Eq. (10). Equation (14) corresponds, after renormalization, to the quark contribution to the condensation energy at $T=\mu=\mu_{H}=0$. Following the renormalization procedure of Ref. [7] we can replace $\Omega_{q} \rightarrow \Omega_{q}^{\text {ren }}$ in Eq. (12), with

$$
\begin{aligned}
\Omega_{q}^{\mathrm{ren}}= & \frac{3 g^{4}}{32 \pi^{2}} N_{c} N_{f} \sigma^{4}-\frac{g^{4} F_{\pi}^{2}}{8 \pi^{2}} N_{c} N_{f} \sigma^{2} \\
& +\frac{g^{4} N_{c} N_{f}}{8 \pi^{2}} \sigma^{4} \log \frac{F_{\pi}}{\sigma} .
\end{aligned}
$$

The finite temperature thermodynamic potential is standard: following Ref. [24] to take into account the helical as well as the number chemical potentials, we get

$\Omega_{T}=-\frac{N_{c} N_{f}}{\beta} \sum_{s= \pm 1} \sum_{\aleph \pm 1} \int \frac{d^{3} p}{(2 \pi)^{3}} \ln \left(1+e^{-\beta\left(E_{p}-s \mu-\aleph \mu_{H}\right)}\right)$,

where $\beta=1 / T$ and the summations run over the signs of energy and helicity. Note that due to the summation over $s$ and $\boldsymbol{\aleph}$ in Eq. (16), the thermodynamic potential at $\mu=0$ and $\mu_{H} \neq 0$ is equivalent to that at $\mu \neq 0$ and $\mu_{H}=0$. This duality has been observed noticed in Ref. [24] for the first time.

For the sake of illustration, we write down the relation between $n_{H}$ and $\mu_{H}, \mu$ and $T$ for the case of an ideal massless gas. This can be obtained analytically by virtue of $\Omega_{T}$ that can be computed exactly in this limit, namely

$$
\begin{aligned}
\frac{\Omega_{T}}{N_{c} N_{f}}= & -\frac{7 \pi^{2} T^{4}}{180}-\frac{\mu^{2} T^{2}}{6}-\frac{\mu^{4}}{12 \pi^{2}} \\
& -\frac{\mu_{H}^{2} T^{2}}{6}-\frac{\mu_{H}^{4}}{12 \pi^{2}}-\frac{\mu_{H}^{2} \mu^{2}}{2 \pi^{2}} .
\end{aligned}
$$


From this relation we get

$$
n_{H}=N_{c} N_{f} \frac{\mu_{H}^{3}}{3 \pi^{2}}+N_{c} N_{f} \mu_{H}\left(\frac{T^{2}}{3}+\frac{\mu^{2}}{\pi^{2}}\right) .
$$

The relation between $n_{H}$ and $\mu_{H}$ is similar to that between $\mu$ and the standard baryonic density:

$$
n_{B}=N_{c} N_{f} \frac{\mu^{3}}{3 \pi^{2}}+N_{c} N_{f} \mu\left(\frac{T^{2}}{3}+\frac{\mu_{H}^{2}}{\pi^{2}}\right) .
$$

The duality $\mu \leftrightarrow \mu_{H}$ is evident by comparing Eqs. (18) and (19).

\section{RESULTS FOR HELICAL MATTER AT ZERO TEMPERATURE}

Our parameter set is $M_{\sigma}=700 \mathrm{MeV}, v=F_{\pi}=93 \mathrm{MeV}$, $M_{\pi}=138 \mathrm{MeV}$ and $g=3.6, \lambda=M_{\sigma}^{2} / 2 F_{\pi}^{2}=28.3$ and $h=M_{\pi}^{2} F_{\pi}=1.78 \times 10^{6} \mathrm{MeV}^{3}$; these give $M_{q}=335 \mathrm{MeV}$ in the vacuum. The thermodynamic potential is invariant under the changes $\mu \rightarrow-\mu$ or $\mu_{H} \rightarrow-\mu_{H}$; therefore, the signs of two chemical potentials are irrelevant and we consider $\mu>0$ and $\mu_{H}>0$ only. Moreover, we have verified that changing $M_{\sigma}$ down to $M_{\sigma}=550 \mathrm{MeV}$ does not alter the results qualitatively; therefore, we limit ourselves to report only the results obtained for $M_{\sigma}=700 \mathrm{MeV}$.

\section{A. The phase diagram at zero temperature}

The phase diagram of a system with unbalanced helicity at $T=0$ has been studied in Ref. [24] for the first time: here we confirm the results of Ref. [24] and in addition to this, we analyze how the vacuum renormalized term of the thermodynamic potential affects the picture. In the upper panel of Fig. 1 we plot the condensate versus $\mu$ at $T=0$ and for several values of $\mu_{H}$. At $\mu_{H}=0$ a first-order phase transition for $\mu=\mu_{c} \approx 365 \mathrm{MeV}$ from the chiral broken phase to the chiral restored phase has been found in agreement with previous model calculations [7]: the chiral restored phase can be dubbed as normal quark matter phase since quark number density is finite while helical density is vanishing. Increasing $\mu_{H}$ we notice that the phase transition becomes smoother and eventually the discontinuity of the order parameter disappears: a smooth crossover replaces the first-order phase transition in some range of $\mu_{H}$. In addition to this, the critical value of $\mu$ becomes smaller. Increasing $\mu_{H}$ even further the crossover becomes stronger again and turns to another first-order phase transition; eventually, at $\mu=0$ and $\mu_{H}=\mu_{c}$ a first-order transition happens to a new phase in which chiral symmetry is restored but quark matter has vanishing density and finite helical density; the fact that $\mu_{c}$ sets the scale for the phase transition in the two cases is due to the duality $\mu \leftrightarrow \mu_{H}$ of the thermodynamic potential.
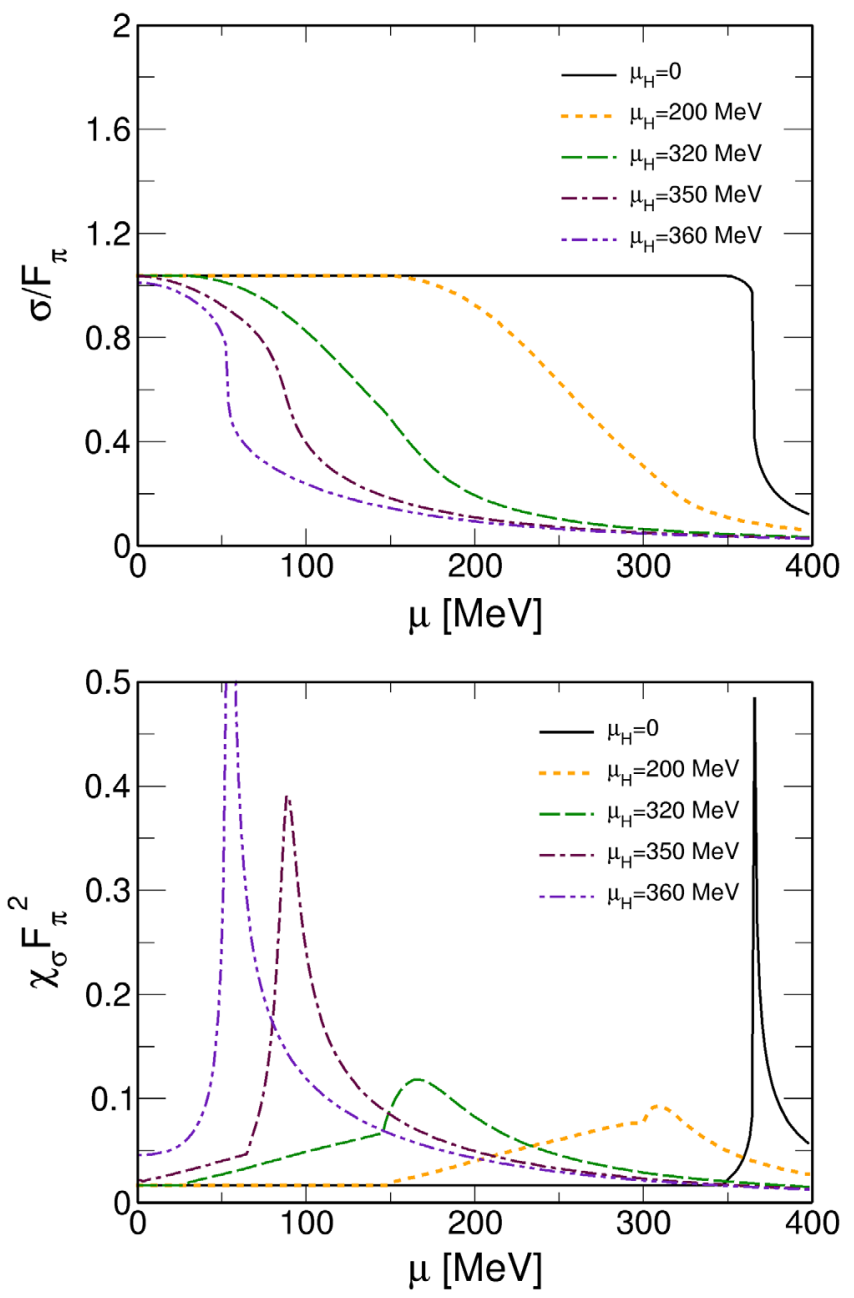

FIG. 1. Condensate (upper panel) and chiral susceptibility (lower panel) versus $\mu$ at $T=0$ and for several values of $\mu_{H}$.

The smoothening followed by the hardening of the phase transitions when $\mu_{H}$ is increased is evident when we compute the chiral susceptibility [53-56]

$$
\chi_{\sigma}=\left(\frac{\partial^{2} \Omega}{\partial \sigma^{2}}\right)^{-1}
$$

around the transitions. The results are shown in the lower panel of Fig. 1 in which we plot the dimensionless quantity $F_{\pi}^{2} \chi_{\sigma}$ versus $\mu$ for several values of $\mu_{H}$. We find that for $\mu \approx \mu_{c}$ or $\mu_{H} \approx \mu_{c}$ the susceptibility is very peaked around the transition, while the peaks become smaller and broader as we move toward the region $\mu \approx \mu_{H}$, signaling that the transition turns to a smooth crossover.

In Fig. 2 we plot the phase diagram in the $\mu-\mu_{H}$ plane at $T=0$. The solid lines denote first-order transitions where a discontinuity of the condensate takes place, while the dashed line stands for the crossover around which the condensate changes smoothly. The diamonds denote the critical end points at which the crossover becomes a 


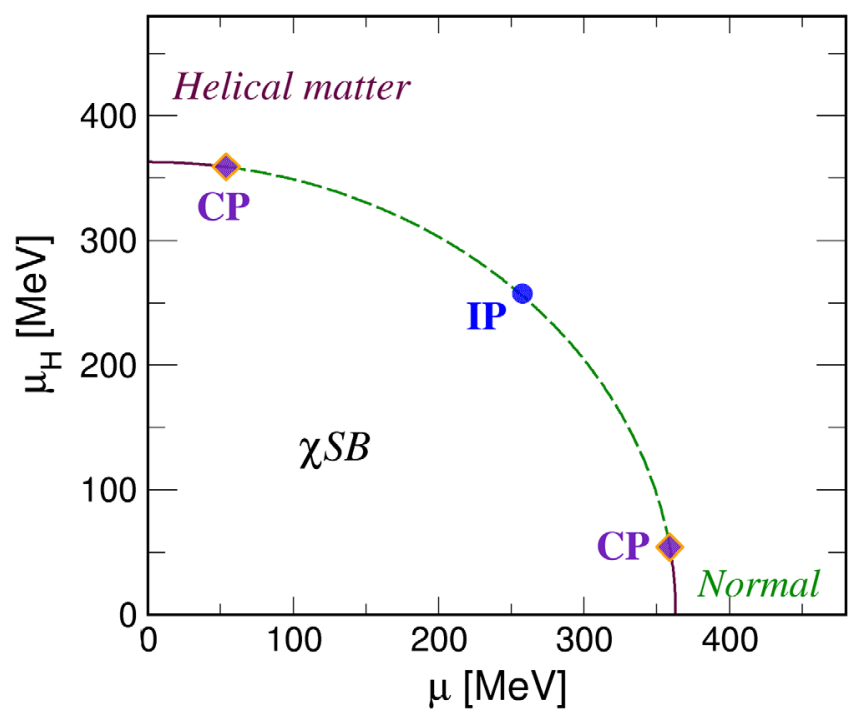

FIG. 2. Phase diagram in the $\mu-\mu_{H}$ plane at $T=0$. Solid lines denote first-order transitions where a discontinuity of the condensate takes place, and the dashed line stands for the crossover around which the condensate changes smoothly; diamonds denote the critical end points $(C P)$ at which the crossover becomes a first-order transition. The blue circle labeled IP stands for the inversion point, namely the point at which the crossover is the smoothest realized in the phase diagram, corresponding to the values of $\mu=\mu_{H}$ at which moving upward or downward along the transition line, the crossover becomes harder. The $\chi \mathrm{SB}$ window denotes the region where chiral symmetry is broken spontaneously; the other regions correspond to phases with finite quark number density $n$ and/or helical density $n_{H}$. In the normal matter chiral symmetry is restored and $n \gg n_{H}$, while helical matter denotes the region where chiral symmetry is restored but $n_{H} \gg n$.

first-order transition. The circle is the inversion point, namely the point at which the crossover is the smoothest realized in the phase diagram, corresponding to the values of $\mu=\mu_{H}$ at which moving upward or downward along the transition line, the crossover becomes harder. All the lines have been obtained from the results shown in Fig. 1 by identifying the critical values of $\mu$ with those at which the chiral susceptibility has the peak. Clearly the transition line is symmetric under the exchange $\mu \leftrightarrow \mu_{H}$. We notice the presence of two critical points in the phase diagram, resulting from the aforementioned duality of the thermodynamic potential. The inversion point (IP) at which the crossover is the smoothest is $\mu=\mu_{H} \approx 257 \mathrm{MeV}$.

The chiral symmetry restored phases in the lower right and upper left corners of the phase diagrams are quite different: for $\mu \gg \mu_{H}$ quark matter has both number and helical densities, denoted by $n$ and $n_{H}$, respectively, but $n \gg n_{H}$; therefore, it is legitimate to call this phase as normal quark matter; on the other corner of the phase diagram where $\mu_{H} \gg \mu$ the dual situation $n_{H} \gg n$ is realized; therefore, this phase can be dubbed as helical quark matter.
One way to distinguish the helical matter from the normal phase is the value of the densities

$$
\begin{gathered}
n=-\frac{\partial \Omega}{\partial \mu}, \\
n_{H}=-\frac{\partial \Omega}{\partial \mu_{H}},
\end{gathered}
$$

where $n$ and $n_{H}$ denote the quark number density and the helical density, respectively. In Fig. 3 we plot $n$ and $n_{H}$ versus the quark chemical potential for two representative values of $\mu_{H}$ : indigo lines correspond to $\mu_{H}=30 \mathrm{MeV}$ to cover the lower right corner of the phase diagram in Fig. 2, and green lines to $\mu_{H}=400 \mathrm{MeV}$ that cover the upper left corner of the phase diagram and extend then to higher values of $\mu$. The normal phase is characterized by $n \gg n_{H}$ while the helical matter has $n_{H} \gg n$. Increasing $\mu$ in the case of large $\mu_{H}$ the system smoothly connects to a phase in which $n \approx n_{H}$.

In Fig. 4 we plot the susceptibilities of quark number density $\chi_{\mu}$ and of helical density $\chi_{\mu H}$, defined, respectively, as

$$
\begin{gathered}
\chi_{\mu}=-\frac{\partial^{2} \Omega}{\partial \mu^{2}}, \\
\chi_{\mu H}=-\frac{\partial^{2} \Omega}{\partial \mu_{H}^{2}} .
\end{gathered}
$$

In the upper panel of Fig. 4 we have collected the results for $\mu_{H}=350 \mathrm{MeV}$, while in the lower panel $\mu_{H}=400 \mathrm{MeV}$ : the two values of $\mu_{H}$ that allow us to probe the helical matter phase crossing the transition line and above the transition line, respectively. We notice that around the crossover line,

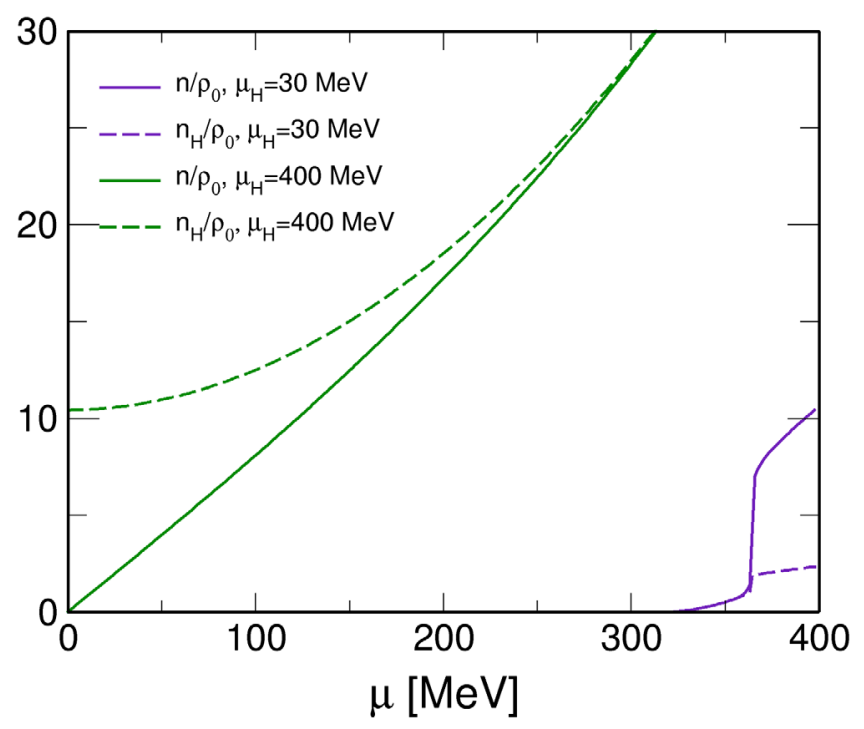

FIG. 3. Densities, measured in units of the nuclear saturation density $\rho_{0}=0.16 \mathrm{fm}^{-3}$, versus $\mu$. 

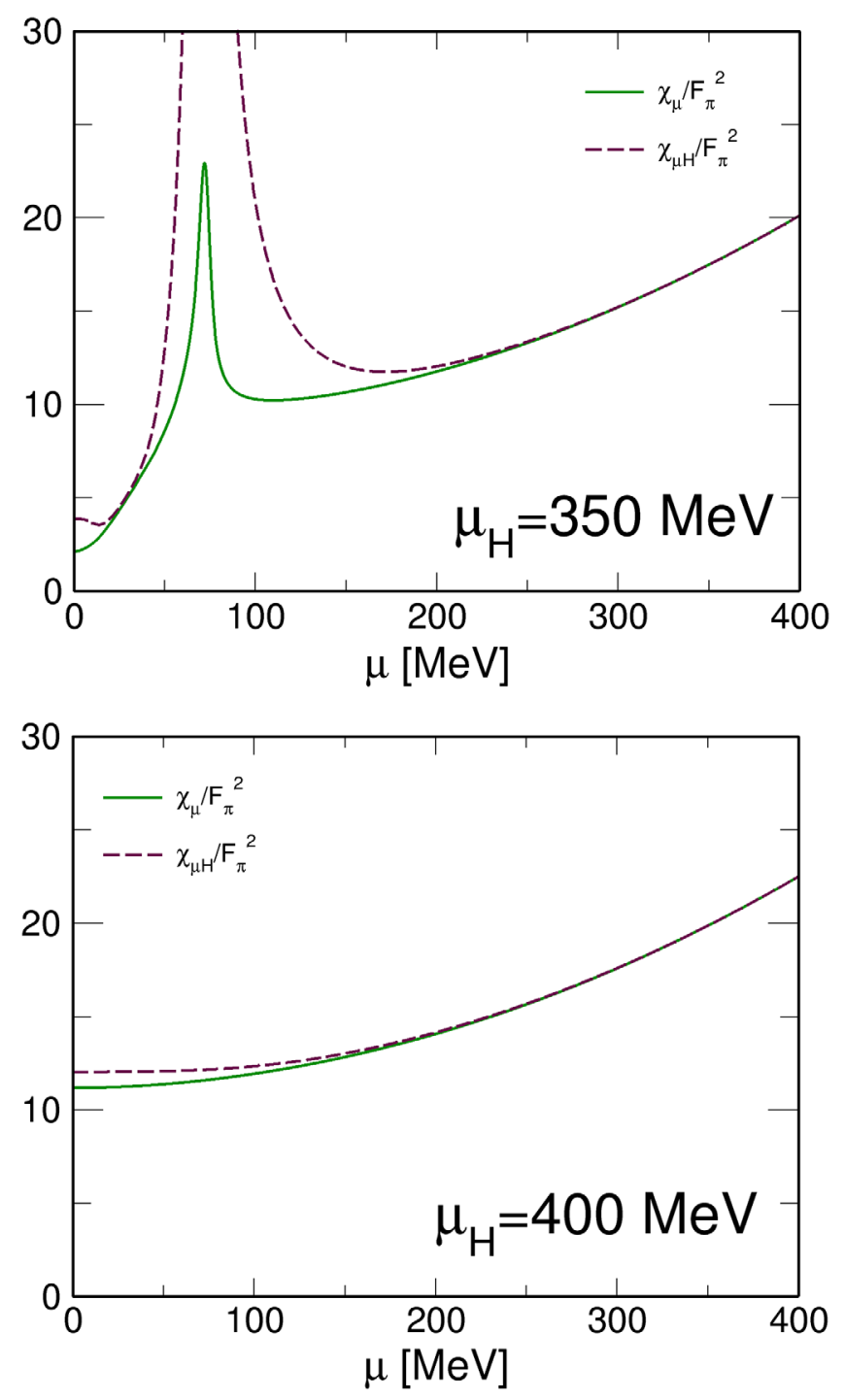

FIG. 4. Condensate (upper panel) and chiral susceptibility (lower panel) versus $\mu$ at $T=0$ and for several values of $\mu_{H}$.

$\chi_{\mu H}$ has a clear peak structure while the peak of $\chi_{\mu}$ is very modest, indicating that in that region the fluctuations of the helical density are much more pronounced that those of the quark number. On the other hand, increasing $\mu$ and getting far from the crossover line, the two susceptibilities agree with each other: quark number and helicity fluctuate in the same way. This is clearer for $\mu_{H}=400 \mathrm{MeV}$ in which case no transition line is crossed: the two susceptibilities agree with each other in the full range of $\mu$ studied, denoting that the fluctuations of the quark number and of the helical density are the same. We have checked that the dual situation happens moving at low $\mu_{H}$ and crossing the critical lines increasing $\mu$.

We have verified that changing the value of $M_{\sigma}$ in the range $(550,700) \mathrm{MeV}$ does not lead to significant changes in the condensates and in the phase diagram. Moreover, lowering the value of $g$ the phase transitions tend to be

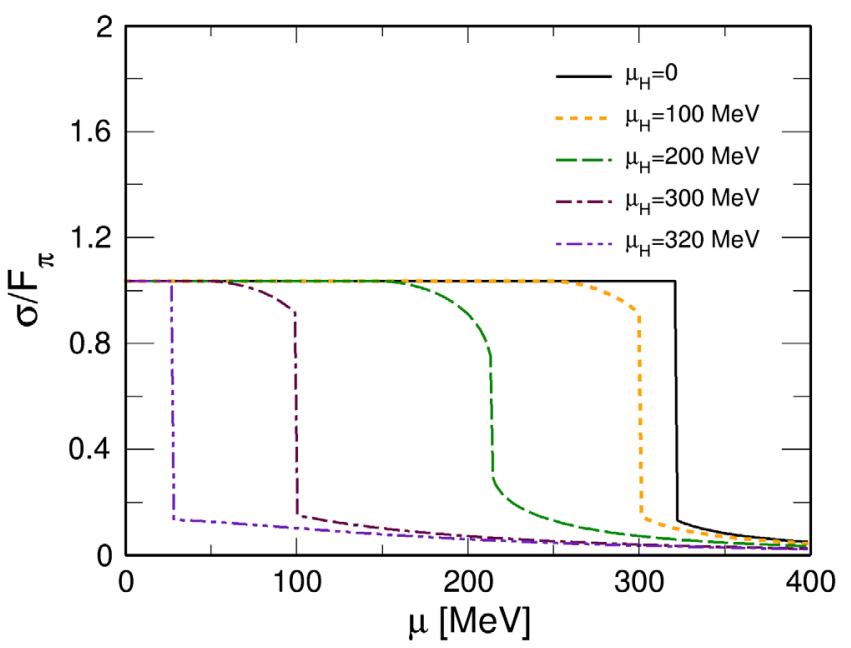

FIG. 5. Condensate versus $\mu$ at $T=0$ and for several values of $\mu_{H}$, computed for the model without the quark renormalized vacuum term in the thermodynamic potential.

smoother and eventually for $M_{q}=g F_{\pi}=300 \mathrm{MeV}$ at $T=\mu=\mu_{H}=0$, the first-order lines disappear.

We have analyzed the effect of removing the renormalized vacuum term of quarks in the thermodynamic potential; see Eqs. (12) and (15). In Fig. 5 we plot the condensate versus $\mu$ at $T=0$ for several values of $\mu_{H}$, for the model without the vacuum term. Firstly, we notice that at $\mu_{H}=0$ the chiral phase transition happens for $\mu=\mu_{c} \approx 320 \mathrm{MeV}$, which is smaller than the critical chemical potential that we have found when we have included the vacuum term; see Fig. 1: the vacuum term makes the chiral symmetry broken phase more stable. Then, we notice that the phase transition remains of the first order for any value of $\mu_{H}$; we notice that changing $\mu_{H}$ from zero to about $200 \mathrm{MeV}$, the jump of the order parameter decreases, and then it increases for larger $\mu_{H}$. We interpret this as a softening of the phase transition for small $\mu_{H}$, which then becomes harder as an inversion point is crossed, similarly to the one in Fig. 2. However, we do not find any range of $\mu_{H}$ in which the transition turns to a smooth crossover: the phase diagram in this case will differ from the one shown in Fig. 2 because in the former case there is only a first-order phase transition line. We conclude that the existence of the critical end points in the zero temperature phase diagram depends on how the regularization of the divergent term of the thermodynamic potential is done; however, the softening followed by the hardening of the phase transition is unaffected by this choice.

\section{RESULTS FOR HELICAL MATTER AT FINITE TEMPERATURE}

\section{A. Chiral symmetry breaking}

In Fig. 6 we plot the $\sigma$ condensate versus temperature, for several values of $\mu$ and $\mu_{H}$. In general, there is a temperature range in which the condensate drops down, signaling 


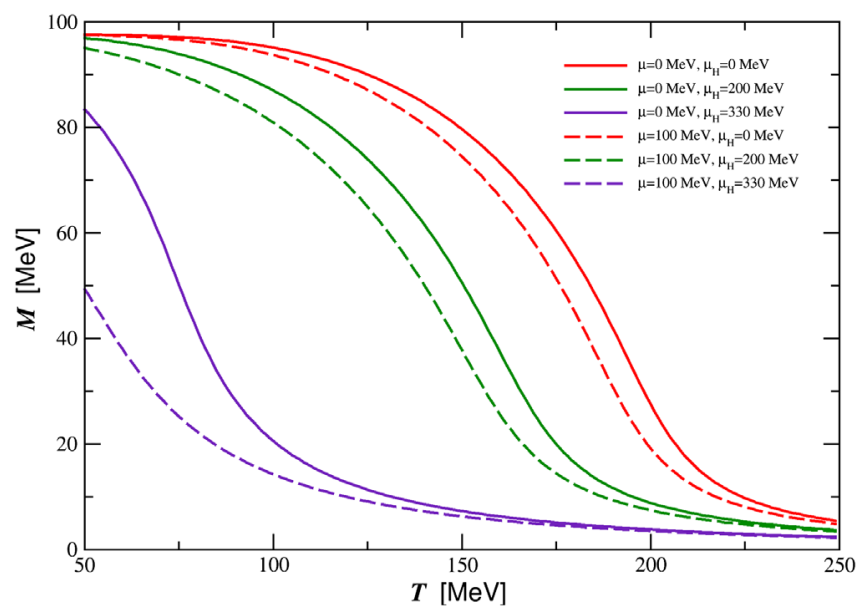

FIG. 6. Constituent quark mass $M$ versus temperature for several $\left(\mu, \mu_{H}\right)$ pairs. Solid and dashed lines correspond to $\mu=$ 0 and $\mu=100 \mathrm{MeV}$, respectively.

the approximate restoration of chiral symmetry via a smooth crossover; the pseudocritical temperature can be identified with the temperature at which $d \sigma / d \beta$ is maximum. Increasing $\mu_{H}$ at $\mu=0$ results in the lowering of the critical temperature. This agrees with the expectations from the zero temperature phase diagram in Fig. 2, since it is shown there that increasing $\mu_{H}$ at $\mu=0$ makes the chiral broken phase less stable and eventually leads to chiral symmetry restoration and helical matter. Therefore, it is expected that increasing $\mu_{H}$ at finite temperature will also lower the $\sigma$ condensate and chiral symmetry restoration will be facilitated. Increasing $\mu$ shifts the pseudocritical temperature to lower values: once again, this is in agreement with the expectation from the zero temperature phase diagram in Fig. 2, because increasing $\mu$ has the effect to destabilize the chiral symmetry broken phase.

\section{B. Phase diagram}

These results can be summarized in a phase diagram in the $\mu-T$ plane; see Fig. 7. The solid lines denote the smooth crossover from the chiral broken phase to the chiral symmetric phase, computed at several values of $\mu_{H}$. Firstly, increasing $\mu_{H}$ moves the critical line downward, in agreement with the previous discussions. The dots in the figures denote the CEP, namely the values of $\mu$ and $T$ at which the crossover becomes a second-order phase transition with divergent susceptibilities; increasing $\mu$ further the critical line becomes first order, with a discontinuity of the $\sigma$ condensate. One of the effects of increasing $\mu_{H}$ is to move the CEP downward; eventually, the CEP disappears from the phase diagram. This agrees with Fig. 2 since at $T=0$ there is a large window in the $\mu-\mu_{H}$ plane in which the transition to helical or normal quark matter happens via a smooth crossover rather than a first-order phase transition.

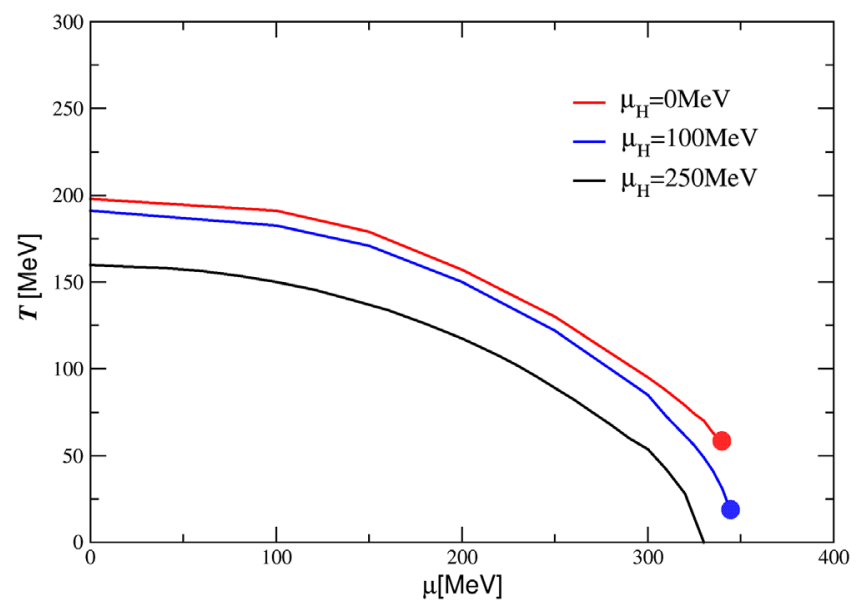

FIG. 7. Pseudocritical lines in the $\mu-T$ plane, for several values of $\mu_{H}$. The dot denotes the critical end point.

\section{Quark number susceptibility}

In Fig. 8 we plot the particle number susceptibility [see Eq. (23)] normalized to temperature, versus temperature, for several values of $\mu$ and $\mu_{H}$. We notice that around the chiral crossover, increasing the value of $\mu_{H}$ results in a broader susceptibility: this becomes evident if we compare the cases $\mu_{H}=200 \mathrm{MeV}$ and $\mu_{H}=0$; see the upper and lower panels in Fig. 8.

The results discussed in this section show that helicity softens the transition from the quark-gluon plasma to the hadron phase. In fact, the helical chemical potential makes the chiral phase transition softer, and if $\mu_{H}$ is large enough, the critical end point disappears from the phase diagram and the transition to the chiral symmetric phase remains a smooth crossover in the whole $\mu-T$ plane. This picture is confirmed by the quark number fluctuations that are smoothed by the helical chemical potential.

\section{HELICAL MATTER UNDER ROTATION}

We close this study by analyzing briefly the correlation between rotation and the fluctuations of the helical density. This investigation is motivated by the potential applications to relativistic heavy ion collisions. As a matter of fact, even if the quark-gluon plasma in collisions was produced with a net $n_{H}=0$, local fluctuations of $n_{H}$ are possible: the amount of fluctuations is measured by the susceptibility $\chi_{\mu H}$ defined in Eq. (24), where the derivative takes into account the dependence of the condensate of $\mu_{H}$. We want to prove that in a rotating medium the fluctuations of $n_{H}$ are enhanced: this in turn would imply that in a medium in local equilibrium, local lumps with $n_{H} \neq 0$ can form and this formation is enhanced if the system is rotating. We limit ourselves to the simplest implementation of rotation of a fermion system; namely, we assume a rigid rotation of an unbounded system with a constant and homogeneous angular velocity $\omega$ directed along the $z$ axis. While the 

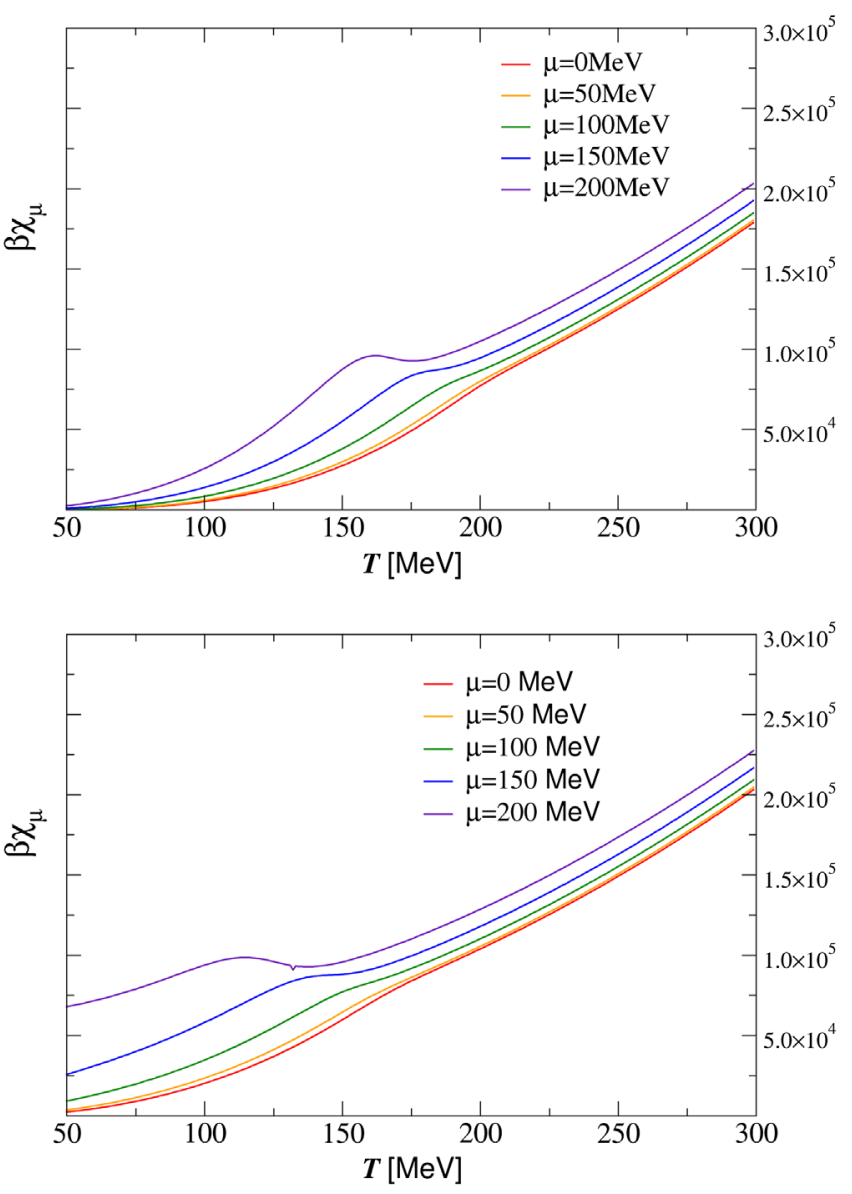

FIG. 8. $\beta \chi_{\mu}$ (in MeV) over temperature, versus temperature, for several values of $\mu$. Upper and lower panel correspond to $\mu_{H}=0$ and $\mu_{H}=200 \mathrm{MeV}$, respectively.

choice of more realistic boundary conditions is feasible $[25,33,34,36]$, these would complicate the calculations but probably would not change qualitatively the results: therefore, in this first study we prefer to adopt the simplest implementation possible to highlight the effect, leaving a complete study to a future article.

The implementation of rotation in quark matter that we adopt has been already discussed several times in the literature; therefore, we quote the results leaving the details to the original references $[25,31-42]$. In previous works a coordinate-dependent condensate has been considered, within the local density approximation in which it is assumed that local equilibrium is realized at any point in space and that the thermodynamic potential per unit volume is given by that of a homogeneous system at any point. Within this approximation, the gap equation for the condensate is solved at each point in space, and then the thermodynamic potential is obtained via an integration over the whole volume. Leaving the solution of the more complete problem to a future study, here we limit ourselves to consider a homogeneous condensate: this approximation is not very bad, considering that even when the coordinate dependence is introduced, on average the condensate varies at most of the $10 \%$ when the radial coordinate $r$ changes from the origin up to $\omega r \lesssim 0.8$. We further limit ourselves to $\mu=0$.

Within the local density approximation, the thermodynamic potential for an unbounded system rotating with angular velocity $\omega$ along the $z$ axis, temperature $T=1 / \beta$ and helical chemical potential $\mu_{H}$ can be obtained from Refs. $[24,36,38,40]$, that is,

$$
\begin{aligned}
\Omega_{M}= & -\frac{N_{c} N_{f}}{4 \beta} \int d^{3} \boldsymbol{r} \sum_{n=-\infty}^{\infty} \int \frac{d p}{8 \pi^{2}} \mathcal{J}_{n}\left(p_{T} r\right) \\
& \times \sum_{s= \pm 1} \sum_{\xi= \pm 1} \sum_{\eta= \pm 1} \log \left(1+e^{\left(s E_{p}+\xi(n+1 / 2) \omega+\eta \mu_{H}\right) \beta}\right),
\end{aligned}
$$

where we have put

$$
\int d p=\int d p_{T}^{2} \int d p_{z}
$$

with $p_{T}$ denoting the transverse momentum while $p_{z}$ is the momentum along the $z$ axis; moreover, $E_{p}=$ $\sqrt{p_{z}^{2}+p_{T}^{2}+(g \sigma)^{2}}$. We have also defined

$$
\mathcal{J}_{n}\left(p_{T} r\right)=J_{n}^{2}\left(p_{T} r\right)+J_{n+1}^{2}\left(p_{T} r\right),
$$

where $J_{n}\left(p_{T} r\right)$ denotes the first kind Bessel function of order $n$. Finally, $\boldsymbol{r}$ is the location from the center of the rotation.

In the form of Eq. (25), $\Omega_{M}$ contains a vacuum part that depends of $\omega$. At difference with previous works [38,40] we subtract this $\omega$-dependent contribution and replace it with our previously renormalized vacuum term; see Sec. II. This procedure is in line with Refs. [34,36] and is justified by the fact that the vacuum term should depend on $\omega$ : an easy way to see this is that in order to experience the rotation, at least one particle should be put on the top of the vacuum so that this particle can feel inertial forces that appear in a rotating system; but the quantum state of vacuum plus one particle is different from the vacuum itself. Therefore, it is not possible to have a vacuum that feels the rotation.

After the subtraction and dividing by the volume of the system, $V$, we are left with the matter part of the thermodynamic potential per unit volume, $\Omega_{T}=\Omega_{M} / V$, that replaces Eq. (16) and reads

$$
\begin{aligned}
\Omega_{T}= & -\frac{N_{c} N_{f}}{2 \beta V} \int d^{3} \boldsymbol{r} \sum_{n=-\infty}^{\infty} \int \frac{d p}{8 \pi^{2}} \mathcal{J}_{n}\left(p_{T} r\right) \\
& \times \sum_{\xi= \pm 1} \sum_{\eta= \pm 1} \log \left(1+e^{-\beta\left(E_{p}+\xi(n+1 / 2) \omega+\eta \mu_{H}\right)}\right) .
\end{aligned}
$$

It is an easy exercise to prove that for $\omega=0 \mathrm{Eq}$. (28) is consistent with Eq. (16). In fact, $\int d^{3} p /(2 \pi)^{3}=\int d p /\left(8 \pi^{2}\right)$ 
for any function that does not depend of the azimuthal angle, and after putting $\omega=0$ in the right-hand side of Eq. (28) the $n$ dependence of the integrand is confined to the $\mathcal{J}_{n}\left(p_{T} r\right)$ term: the summation over $n$ can be performed by using $J_{-n}=(-1)^{n} J_{n}$ as well as the well-known identity

$$
J_{0}^{2}(x)+2 \sum_{n=1}^{\infty} J_{n}^{2}(x)=1,
$$

which brings an overall factor of 2 in Eq. (28).

Under the assumption that the condensate does not depend of $\boldsymbol{r}$, the integration over volume can be done exactly: considering that $\mathcal{J}_{n}=\mathcal{J}_{|n|-1}$ for $n<0$ we can limit ourselves to evaluate $\mathcal{J}_{n}$ for $n>0$. We take a cylinder with radius $R$ and height $h$ that develops along the direction of $\omega$ : we have $V=\pi R^{2} h$ and

$$
\begin{aligned}
\int d^{3} \boldsymbol{r} \mathcal{J}_{n}\left(p_{T} r\right)= & 2 V\left[J_{n}^{2}\left(p_{T} R\right)+J_{n+1}^{2}\left(p_{T} R\right)\right. \\
& \left.-\frac{(2 n+1)}{p_{T} R} J_{n}\left(p_{T} R\right) J_{n+1}\left(p_{T} R\right)\right], \\
n \geq & 0 .
\end{aligned}
$$

We limit ourselves to describe a region that is very close to the center of the rotation, putting $p_{T} R=0$ in the argument of $\mathcal{J}_{n}$ in Eq. (28). For $x \approx 0$ the Bessel functions behave as $J_{0}^{2}(x) \approx 1, J_{n}^{2}(x) \approx x^{2|n|}$ for $n \neq 0$; in this approximation, we have to consider only the contribution from $J_{0}$ and thus take into account $\mathcal{J}_{0}=\mathcal{J}_{-1} \approx 1$ in the sum over $n$ in Eq. (28): the integration in Eq. (30) gives $V$ for $n=-1,0$ and the approximate $\Omega_{T}$ is

$$
\begin{aligned}
\Omega_{T}= & -\frac{N_{c} N_{f}}{\beta} \int \frac{d p}{8 \pi^{2}} \\
& \times \sum_{\kappa= \pm 1 / 2} \sum_{\eta= \pm 1} \log \left(1+e^{-\beta\left(E_{p}+\kappa \omega+\eta \mu_{H}\right)}\right) .
\end{aligned}
$$

In the following we use Eq. (31) to estimate the effect of $\omega \neq 0$ on the fluctuations of the helical density: firstly, we illustrate the idea with an ideal gas of massive particles; then, we compute $\chi_{H}$ for the QM model.

\section{A. The case of an ideal gas}

It is instructive to evaluate the effect of $\omega$ on $\chi_{H}$ in the case of an ideal gas with massive particles with mass $m$. In this case the thermodynamic potential is given by Eq. (28) only.

In order to mimic the conditions of high-energy nuclear collisions we consider $\mu_{H} \approx 0$. Moreover, to make the coupling between $\omega$ and $\mu_{H}$ more transparent, we consider the lowest nontrivial order in $\omega$ in the expansion of $\Omega_{T}$ around $\mu_{H}=0$ and $\omega=0$. Considering that $\Omega_{T}$ is an even function of $\mu_{H}$ and $\omega$ we are left with

$$
\begin{aligned}
\Omega_{T}= & \Omega_{T}\left(\mu_{H}=0, \omega=0\right) \\
& +\frac{c_{2,0}}{2} \mu_{H}^{2}+\frac{c_{0,2}}{2} \omega^{2}+\frac{c_{4,0}}{4 !} \mu_{H}^{4}+\frac{c_{0,4}}{4 !} \omega^{4} \\
& +\frac{c_{2,2}}{4} \omega^{2} \mu_{H}^{2}+O\left(\mu_{H}^{6}, \omega^{6}\right),
\end{aligned}
$$

where

$$
c_{m, n}=\left.\frac{\partial^{(m+n)} \Omega_{T}}{\partial \mu_{H}^{m} \partial \omega^{n}}\right|_{\mu_{H}=0, \omega=0} .
$$

At the lowest nontrivial order the coupling between $\mu_{H}$ and $\omega$ is given by the term proportional to $c_{2,2}$ in Eq. (32).

The fluctuations of the helical density are described by $\chi_{\mu H}=-\partial^{2} \Omega_{T} / \partial \mu_{H}^{2}$; from Eq. (32) at $\mu_{H} \approx 0$ we read easily

$$
\chi_{\mu H}=-c_{2,0}-\frac{c_{2,2}}{2} \omega^{2} .
$$

Within these approximations we can embed the effects of rotation on $\chi_{\mu H}$ into the dimensionless coefficient $c_{2,2}$.

In the massless case $c_{2,0}$ and $c_{2,2}$ can be computed analytically with the result

$$
\begin{gathered}
c_{2,0}=-\frac{N_{c} N_{f}}{3} T^{2}, \\
c_{2,2}=-\frac{N_{c} N_{f}}{2 \pi^{2}} .
\end{gathered}
$$

Therefore in the massless limit we can write

$$
\chi_{\mu H}=\frac{N_{c} N_{f}}{3} T^{2}+\frac{N_{c} N_{f}}{4 \pi^{2}} \omega^{2} .
$$

Note that the enhancement of fluctuations of helical density $\propto \omega^{2}$ in the above equation is not related to the restoration of chiral symmetry but to the fact that quark matter rotates. A similar rotation can be found for the susceptibility of the baryonic density.

For $m \neq 0$ the calculation of $c_{2,2}$ has to be done numerically. We show $c_{2,2}$ versus temperature in Fig. 9 for several values of $m$. Note that $c_{2,2}<0$ which means that $\omega \neq 0$ enhances the fluctuations of the helical density at a given temperature. This happens because $\omega$ modifies the single-particle spectrum like a chemical potential; see Eq. (31).

\section{B. The case of the QM model}

We limit ourselves to compute $\chi_{H}$ for $\mu=\mu_{H}=0$ to mimic the conditions of the medium produced in very-highenergy nuclear collisions. We write $\Omega=U+\Omega_{q}^{\text {ren }}+\Omega_{T}$. The thermal part of the thermodynamic potential is given by Eq. (31) while the classical potential and the renormalized vacuum term are given by Eqs. (13) and (15), respectively. 


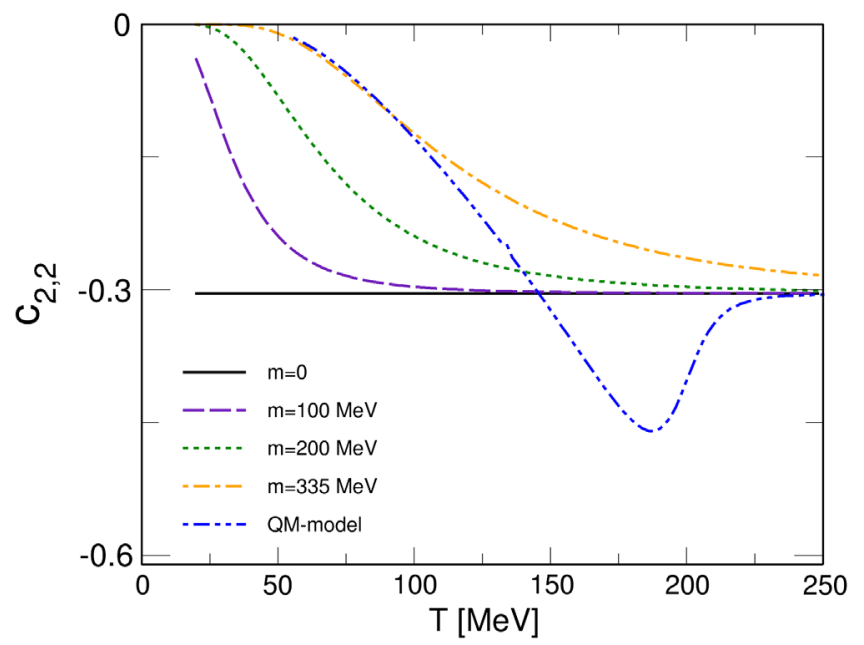

FIG. 9. Coefficient $c_{2,2}$ versus temperature, for several values of the quark mass $m$ and for the QM model.

We firstly show the coefficient $c_{2,2}$ in Fig. 9. This has been computed by numerical differentiation of $\Omega$ similarly to Eq. (33):

$$
c_{2,2}=\left.\frac{d^{4} \Omega}{d \mu_{H}^{2} d \omega^{2}}\right|_{\mu_{H}=0, \omega=0} .
$$

At difference with Eq. (33), we have used the total derivative notation in Eq. (38) to emphasize that the differentiation takes into account of the $\omega$ and $\mu_{H}$ dependence of the condensate. $c_{2,2}$ is negative in agreement with the discussion for the ideal gas, signaling the enhancement of the fluctuations induced by a nonzero $\omega$. The most striking difference between the QM model and the fixed mass results is that in the former case the chiral phase transition affects $c_{2,2}$, as it is clear from the groove structure around $T_{c}$. Thus, in magnitude the enhancement

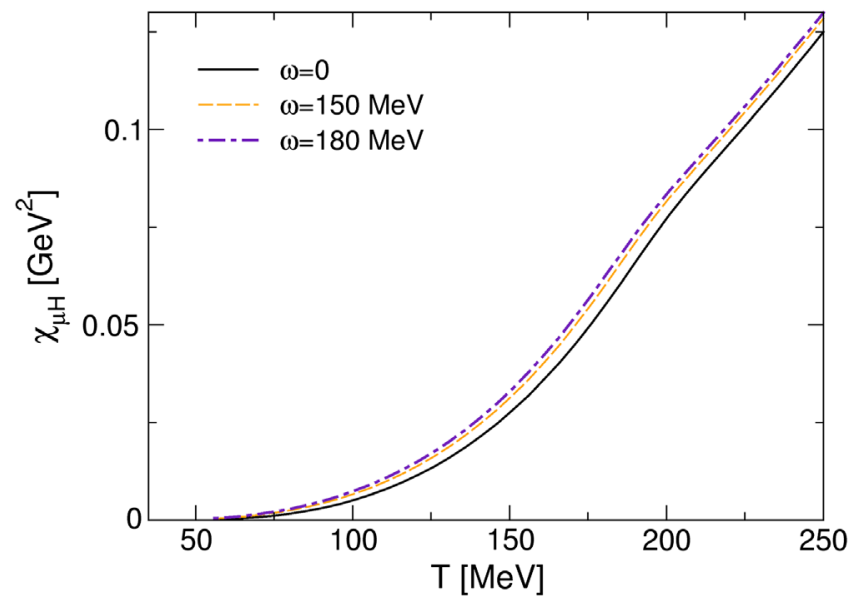

FIG. 10. $\chi_{\mu H}$ versus temperature, for several values of $\omega$ at $\mu_{H}=0$. of fluctuations due to $\omega \neq 0$ is larger than the one obtained for an ideal gas.

We complete this study by computing $\chi_{\mu H}$ versus temperature for $\omega \neq 0$ within the QM model. The results are summarized in Fig. 10. The enhancement of the susceptibility induced by $\omega$ is evident. We conclude that a rigid rotation enhances the fluctuations of helical density.

\section{CONCLUSIONS AND OUTLOOK}

We have studied the thermodynamics of quark matter with a helical chemical potential $\mu_{H}$ conjugated to helical density $n_{H}$, together with the standard baryon chemical potential $\mu$. We have analyzed chiral symmetry restoration as well as several susceptibilities of helical matter within the renormalized quark-meson model with two flavors of quarks.

Helicity is a conserved quantity for free massive and massless quarks; within the model at hand, the interaction among quarks has the effect to shift the mass from the current to the constituent one, giving a Hamiltonian consistent with that of a massive Dirac particle which justifies the use of the helicity as a good quantum number. Thinking of potential applications of the material discussed here to relativistic heavy ion collisions, we mention that despite the large scattering rate that gives a low shear viscosity over entropy density ratio, the relaxation time of helicity in the hot quark-gluon plasma has been estimated to be larger than the lifetime of the quark-gluon plasma produced in the collisions. For these reasons, it is meaningful to consider the helical density $n_{H}$ as an approximately conserved quantity in heavy ion collisions, which allows us to formulate helical density fluctuations in terms of $\mu_{H}$.

We have computed the phase diagram at zero temperature: the result is summarized in Fig. 2. We have found a critical line in the $\mu-\mu_{H}$ plane which contains two critical end points. The middle portion of this line is made of a smooth crossover, while the lower right and upper left corners of the line are first-order phase transitions. The symmetry of the phase diagram around the line $\mu=\mu_{H}$ can be understood easily in terms of the duality of the thermodynamic potential for the exchange $\mu \leftrightarrow \mu_{H}$. We have characterized the transition from the hadron gas at $T=0$ to the helical matter in terms of average helical and baryonic density $n_{B}$; in particular, for helical matter $n_{H} \gg n_{B}$. We have also considered the fluctuations of helical and baryonic density. When the transition at large $\mu_{H}$ is considered, the fluctuations of $n_{H}$ are enhanced in comparison to those of $n$. This might be an additional difference, besides $n_{H} \gg n_{B}$, between normal and helical quark matter.

We have then studied chiral symmetry restoration at finite temperature. The helical chemical potential disfavors chiral symmetry breaking and thus leads to a lower pseudocritical line. We have completed the study by 
computing the particle number susceptibilities around the chiral crossover. Overall, our results support the idea that $\mu_{H}$ makes the chiral phase transition softer and eventually for large values of the chemical potential, the chiral critical end point disappears from the $T-\mu$ diagram. Our results at zero as well as finite temperature are in agreement with Ref. [24].

We have also examined briefly the role of a rigid rotation on the fluctuations of helical density. To mimic the conditions of the quark medium produced in very-highenergy nuclear collisions we have analyzed this problem for the case $\mu=\mu_{H}=0$. For the sake of simplicity, and since this is the first time in which this problem has been studied, we have used the simplest implementation of the rotation of quark matter at finite temperature by modeling an unbounded system in local equilibrium, limiting ourselves to study the region close to the rotation axis. The susceptibility of the helical density $\chi_{\mu H}$ is enhanced by the rotation. We have illustrated this idea for an ideal gas, then analyzing the same problem within the quark-meson model. Firstly, we have embedded the effect of rotation on $\chi_{\mu H}$ into a dimensionless coefficient $c_{2,2}$, shown in Fig. 9 and computed analytically for the massless case; see Eqs. (36) and (37). Then we have done the computation of this coefficient for the quark-meson model, finding that it is sensitive to the restoration of chiral symmetry at finite temperature. Finally, for the QM model we have presented the full calculation of $\chi_{\mu H}$. Our conclusion is that a rigid rotation enhances the fluctuations of $n_{H}$ in a system in thermodynamic equilibrium. Therefore, even though a medium with a net $n_{H}=0$ is formed in high-energy nuclear collisions, lumps of matter with $\left\langle n_{H}^{2}\right\rangle \neq 0$ can form because of event-by-event fluctuations, and this formation is favored in rotating matter. A more detailed study of rotation, including proper boundary conditions, will be the subject of a forthcoming article.

Altogether, our result could have some impact on relativistic heavy ion collisions: in fact, lumps of matter with $\left\langle n_{H}^{2}\right\rangle \neq 0$ can be produced due to event-by-event fluctuations. Thus, it is meaningful to question about the effect of helical density in the collisions. We have found that helical density makes the chiral phase transition smoother and lowers the critical temperature: it is likely that the local production of $n_{H}$ by fluctuations lowers the freeze-out temperature. In addition to this, our model calculations show that the chiral phase transition is smoother in the lumps with $\left\langle n_{H}^{2}\right\rangle \neq 0$, and this can affect the observables that are sensitive to the location of the critical end point in the $T-\mu$ plane.

Other tests of the present work would consist in the implementation of fluctuations of the helical density of the quark-gluon plasma, in numerical codes based on relativistic transport theory in which spin dynamics is included. Then, it would be possible to compute the effect of these fluctuations on some final state observables, like hadron spectra, collective flows and many-particle correlators. Along these lines, there are already preliminary studies that suggest that spin dynamics might be important to explain the $\Lambda$ polarization puzzle [29]. We might use the aforementioned codes to study this problem in a more systematic way, having the advantage to use a solid numerical setup that is already capable to reproduce many observables of heavy ion collisions. If the $\Lambda$ polarization that turns out from these new calculations was not in agreement with the measured one, then we could argue that helical fluctuations are not so relevant in heavy ion collisions.

This study can be improved by introducing the charge chemical potential, that would split the chemical potential of up and down quarks. This would be useful to study charge fluctuations in heavy ion collisions.

While a complete study of the phenomenological impact of helical density on observables of heavy ion collisions is well beyond the purpose of the present article, our results suggest that potentially helical matter can affect the evolution of the medium created by the collisions, and we plan to report on this topic in the future.

\section{ACKNOWLEDGMENTS}

The authors acknowledge discussions with Roberto Anglani, Maxim Chernodub, Marco Frasca and Stefano Nicotri. M. R. acknowledges John Petrucci for inspiration. The work of the authors is supported by the National Science Foundation of China (Grants No. 11805087 and No. 11875153).
[1] S. Borsanyi, Z. Fodor, C. Hoelbling, S. D. Katz, S. Krieg, C. Ratti, and K. K. Szabó (Wuppertal-Budapest Collaboration), J. High Energy Phys. 09 (2010) 073.

[2] S. Borsanyi, G. Endrodi, Z. Fodor, A. Jakovac, S. D. Katz, S. Krieg, C. Ratti, and K. K. Szabo, J. High Energy Phys. 11 (2010) 077.
[3] M. Cheng et al., Phys. Rev. D 81, 054504 (2010).

[4] A. Bazavov et al., Phys. Rev. D 85, 054503 (2012).

[5] S. Borsanyi, Z. Fodor, C. Hoelbling, S. D. Katz, S. Krieg, and K. K. Szabo, Phys. Lett. B 730, 99 (2014).

[6] V. Skokov, B. Friman, E. Nakano, K. Redlich, and B. J. Schaefer, Phys. Rev. D 82, 034029 (2010). 
[7] B. Zhang, S. S. Wan, and M. Ruggieri, Phys. Rev. D 101, 016014 (2020).

[8] M. Frasca and M. Ruggieri, Phys. Rev. D 83, 094024 (2011).

[9] M. Ruggieri, M. Tachibana, and V. Greco, J. High Energy Phys. 07 (2013) 165.

[10] M. Ruggieri, L. Oliva, P. Castorina, R. Gatto, and V. Greco, Phys. Lett. B 734, 255 (2014).

[11] T. K. Herbst, J. M. Pawlowski, and B. J. Schaefer, Phys. Lett. B 696, 58 (2011).

[12] R. Khan, J. O. Andersen, L. T. Kyllingstad, and M. Khan, Int. J. Mod. Phys. A 31, 1650025 (2016).

[13] Y. Nambu and G. Jona-Lasinio, Phys. Rev. 122, 345 (1961).

[14] Y. Nambu and G. Jona-Lasinio, Phys. Rev. 124, 246 (1961).

[15] U. Vogl and W. Weise, Prog. Part. Nucl. Phys. 27, 195 (1991).

[16] S. P. Klevansky, Rev. Mod. Phys. 64, 649 (1992).

[17] T. Hatsuda and T. Kunihiro, Phys. Rep. 247, 221 (1994).

[18] M. Buballa, Phys. Rep. 407, 205 (2005).

[19] M. Asakawa and K. Yazaki, Nucl. Phys. A504, 668 (1989).

[20] H. Zhang, D. Hou, T. Kojo, and B. Qin, Phys. Rev. D 96, 114029 (2017).

[21] R. Câmara Pereira, R. Stiele, and P. Costa, Eur. Phys. J. C 80, 712 (2020).

[22] G. G. Barnafoldi, A. Jakovac, and P. Posfay, Phys. Rev. D 95, 025004 (2017).

[23] R. A. Tripolt, B. J. Schaefer, L. von Smekal, and J. Wambach, Phys. Rev. D 97, 034022 (2018).

[24] M. N. Chernodub and V.E. Ambrus, arXiv:2005.03575 [Phys. Rev. D (to be published)].

[25] V. E. Ambrus, J. High Energy Phys. 08 (2020) 016.

[26] V. E. Ambrus and M. N. Chernodub, arXiv:1912.11034.

[27] P. B. Pal, Am. J. Phys. 79, 485 (2011).

[28] J. I. Kapusta, E. Rrapaj, and S. Rudaz, Phys. Rev. C 101, 031901 (2020).

[29] V. E. Ambrus and M. N. Chernodub, arXiv:2010.05831.

[30] C. Manuel and J. M. Torres-Rincon, Phys. Rev. D 92, 074018 (2015).

[31] Y. Jiang, Z. W. Lin, X. G. Huang, and J. Liao, EPJ Web Conf. 171, 07004 (2018).

[32] H. L. Chen, K. Fukushima, X. G. Huang, and K. Mameda, Phys. Rev. D 93, 104052 (2016).
[33] S. Ebihara, K. Fukushima, and K. Mameda, Phys. Lett. B 764, 94 (2017).

[34] M. N. Chernodub and S. Gongyo, J. High Energy Phys. 01 (2017) 136.

[35] B. R. Iyer, Phys. Rev. D 26, 1900 (1982).

[36] V. E. Ambrus and E. Winstanley, Phys. Rev. D 93, 104014 (2016).

[37] A. Yamamoto and Y. Hirono, Phys. Rev. Lett. 111, 081601 (2013).

[38] Y. Jiang and J. Liao, Phys. Rev. Lett. 117, 192302 (2016).

[39] A. Vilenkin, Phys. Rev. D 21, 2260 (1980).

[40] X. Wang, M. Wei, Z. Li, and M. Huang, Phys. Rev. D 99, 016018 (2019).

[41] A. L. Fetter, Rev. Mod. Phys. 81, 647 (2009).

[42] V.E. Ambruş and E. Winstanley, Phys. Lett. B 734, 296 (2014).

[43] Z. T. Liang and X. N. Wang, Phys. Rev. Lett. 94, 102301 (2005); 96, 039901(E) (2006).

[44] X. G. Huang, P. Huovinen, and X. N. Wang, Phys. Rev. C 84, 054910 (2011).

[45] X. G. Huang, Rep. Prog. Phys. 79, 076302 (2016).

[46] F. Becattini and F. Piccinini, Ann. Phys. (Amsterdam) 323, 2452 (2008).

[47] F. Becattini, V. Chandra, L. Del Zanna, and E. Grossi, Ann. Phys. (Amsterdam) 338, 32 (2013).

[48] F. Becattini, G. Inghirami, V. Rolando, A. Beraudo, L. Del Zanna, A. De Pace, M. Nardi, G. Pagliara, and V. Chandra, Eur. Phys. J. C 75, 406 (2015); 78, 354(E) (2018).

[49] Y. Jiang, Z. W. Lin, and J. Liao, Phys. Rev. C 94, 044910 (2016); 95, 049904(E) (2017).

[50] A. Aristova, D. Frenklakh, A. Gorsky, and D. Kharzeev, J. High Energy Phys. 10 (2016) 029.

[51] W. T. Deng and X. G. Huang, Phys. Rev. C 93, 064907 (2016).

[52] M. Mannarelli, Particles 2, 411 (2019).

[53] K. Fukushima, Phys. Lett. B 591, 277 (2004).

[54] C. Sasaki, B. Friman, and K. Redlich, Phys. Rev. D 75, 074013 (2007).

[55] C. Sasaki, B. Friman, and K. Redlich, Phys. Rev. D 75, 054026 (2007).

[56] H. Abuki, R. Anglani, R. Gatto, G. Nardulli, and M. Ruggieri, Phys. Rev. D 78, 034034 (2008). 